CASE REPORT

\title{
Gonococcal endocarditis: a rare complication of a common disease
}

\author{
A Shetty, D Ribeiro, A Evans, S Linnane
}

J Clin Pathol 2004;57:780-781. doi: 10.1136/icp.2003.015628

This report describes a case of gonococcal endocarditis in a 28 year old male patient with no history of previous valvular heart disease. More cases of gonococcal endocarditis (a rare complication of gonorrhoea) may occur with the increase in the incidence of gonorrhoea and the increase in resistance to ciprofloxacin, which is currently used as the first line agent for the treatment of gonorrhoea in $75 \%$ of genitourinary medicine clinics.

W ith resurgence in the incidence of gonorrhoea in the UK we are likely to see more instances of its complications being manifested. Furthermore, the increase in resistance to ciprofloxacin (the GRASP study revealed a 7\% increase in ciprofloxacin resistance in England and Wales from 2001 to 2002), ${ }^{1}$ which is used as a first line agent against gonorrhoea could mean more patients being inadequately treated, giving rise to a greater risk of disseminated infections. We present a case of gonococcal endocarditis, which is one of the rare complications of gonorrhoea.

\section{CASE REPORT}

In February 2003 a 28 year old male patient presented to his local general practitioner with symptoms including sore throat, lethargy, general malaise, arthralgia, particularly of the knees, sweating, and fevers mainly at night. His general practitioner found no specific abnormalities on thorough general examination and prescribed a course of amoxicillin $500 \mathrm{mg}$ three times a day for 10 days.

His symptoms failed to respond to this treatment and his night sweats worsened. He re-presented to his general practitioner, who noticed the development of a new grade 3, pansystolic murmur at the apex, which radiated to the axilla. Admission to hospital was arranged.

On admission the murmur was confirmed. The patient was found to have clubbing of the fingernails and a vasculitic lesion on the left great toe. Investigation showed a neutrophilic leucocytosis $\left(18 \times 10^{9} /\right.$ litre $)$, a raised $\mathrm{C}$ reactive protein (> $120 \mathrm{mg} /$ litre), and a raised erythrocyte sedimentation rate ( $51 \mathrm{~mm} /$ hour). Liver function tests showed a reduced serum albumin at $29 \mathrm{~g}$ /litre (normal range, 35-45). An initial diagnosis of bacterial endocarditis was made. Four separate sets of blood cultures were obtained before intravenous empirical treatment with benzylpenicillin ( $1.2 \mathrm{~g}$ at four hourly intervals) and gentamicin ( $80 \mathrm{mg}$ twice daily) was initiated.

Transthoracic echocardiography revealed a large echodense mass ( $1.35 \mathrm{~cm}$ diameter) on the anterior mitral valve leaflet, with associated severe mitral regurgitation. Left ventricular function was unimpaired.

On day four of his admission, blood culture results became available; the aerobic bottles of all three sets had grown a Gram negative diplococcus, identified as Neisseria gonorrhoeae serotype WI. The organism was $\beta$ lactamase negative, with a minimum inhibitory concentration of $0.03 \mu \mathrm{g} / \mathrm{ml}$ to penicillin, and was resistant to ciprofloxacin. Admission antibiotics were continued.

The patient gave no history of previous venereal disease or intravenous drug abuse. He had had two sexual partners between December 2002 and February 2003, one of whom had received treatment for genitourinary symptoms. He felt his symptoms had begun several days after unprotected sexual contact with this individual. Chlamydia and syphilis serology were both negative.

By the seventh inpatient day, the white cell count had fallen to $11 \times 10^{9}$ /litre and his $C$ reactive protein to $53 \mathrm{~g} /$ litre. However, he continued to have recurrent fevers and his erythrocyte sedimentation rate was still raised at $85 \mathrm{~mm} /$ hour. He was subsequently transferred from the general medical to the cardiology services for further management.

A transoesophageal echocardiogram was performed, 17 days after admission, which confirmed the findings of the transthoracic echocardiogram, but revealed no evidence of paravalvular abscess formation. Because of failure to improve, a change of antibiotic treatment was implemented on the same day; gentamicin was stopped and replaced with rifampicin at a dose of $600 \mathrm{mg}$ daily. On day 19 the patient developed severe nausea, which was attributed to the benzylpenicillin. This was replaced with cefotaxime at a dose of $2 \mathrm{~g}$ at six hourly intervals. This had no appreciable effect on his condition, his inflammatory markers did not settle, and he remained pyrexial. On day 25 , a decision was made to proceed to mitral valve replacement because medical treatment was deemed to have been unsuccessful (fig l).

At surgery, the native mitral valve was replaced with a $29 \mathrm{~mm}$ On-X bileaflet mechanical prosthetic valve. Culture and Gram stain of the native valve failed to show evidence of $N$ gonorrhoeae. He completed a two week course of intravenous cefotaxime and was discharged, well, on warfarin 38 days after admission. He will require ongoing cardiology follow up.

\section{DISCUSSION}

Disseminated gonococcal infection (DGI) occurs in $1-3 \%$ of all gonococcal infections and gonococcal endocarditis is a complication in $1-2 \%$ of patients with DGI. ${ }^{2}$ In the worldwide literature, there are 120 reported cases in the pre-antibiotic era and 50 cases in the post-antibiotic era. Most of the cases in the post-antibiotic era have occurred in the 1980s and 1990s, suggesting a trend towards an increase in this rare complication of gonorrhoea. ${ }^{2}$

The duration from the onset of symptoms to diagnosis is around 45 days, which is longer than the acute presentation of staphylococcal and pneumococcal endocarditis, but shorter than the subacute presentation of viridans streptococcal endocarditis. It occurs more often in younger age groups (15-35 years) and is more common in men than women, although DGI occurs more often in women. Genitourinary

Abbreviations: $\mathrm{DGl}$, disseminated gonococcal infection 


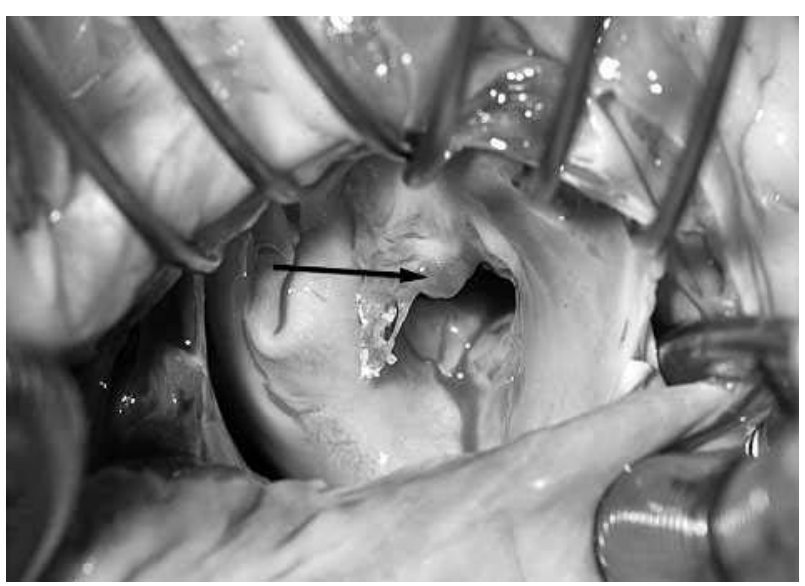

Figure 1 Intraoperative picture with the large vegetation on the mitral valve leaflet.

symptoms are seen in only a third of patients. Double quotidian fever (two distinct spikes of fever in one day) was regarded as a characteristic feature in the pre-antibiotic era, but is rarely seen now. ${ }^{2}$

"More than half of the patients with gonococcal endocarditis require valve surgery, which is usually as a result of worsening congestive cardiac failure"

The vegetations are usually large and are picked up on echocardiography in about $90 \%$ of cases. ${ }^{2}$ The aortic valve is most commonly affected. Compared with many other causes of bacterial endocarditis, a history of pre-existing valvular heart disease is unusual (13\% of cases compared with $86 \%$ of cases of viridans streptococci endocarditis). ${ }^{3}$ A worrying feature is that the patient may acutely deteriorate after weeks of appropriate antibiotic treatment. More than half of the patients with gonococcal endocarditis require valve surgery, which is usually as a result of worsening congestive cardiac
Take home messages

- We report a case of gonococcal endocarditis in a 28 year old man with no history of previous valvular heart disease

- With the rise in the diagnoses of gonorrhoea and increase in ciprofloxacin resistance we are likely to see more disseminated infections, including rare complications such as endocarditis

failure. The mortality rate is alarmingly high at 19\%, despite appropriate medical and surgical treatment. ${ }^{2}$

\section{ACKNOWLEDGEMENTS}

Dr N Masani and Mr P O'Keefe, University Hospital of Wales, Cardiff, UK.

\section{Authors' affiliations}

A Shetty, D Ribeiro, Department of Microbiology, University Hospital of Wales, No 62, Heol Y Cadno, Thornhill, Cardiff CF 14 9DY, UK

A Evans, S Linnane, Llandough Hospital, Penlan Road, Penarth Vale of Glamorgan, CF64 2XX, UK

Correspondence to: Dr A Shetty, Department of Microbiology, University Hospital of Wales, No 62, Heol Y Cadno, Thornhill, Cardiff CF 14 9DY, UK; anjalishettyuk@yahoo.co.uk

Accepted for publication 31 January 2004

\section{REFERENCES}

1 Fenton KA, Ison C, Johnson AP, et al. Ciprofloxacin resistance in Neisseria gonorrhoeae in England and Wales in 2002. Lancet 2003;361:1867-9.

2 Jackman JD Jr, Glamann DB. Southwestern internal medicine conference: gonococcal endocarditis: twenty-five year experience. Am J Med Sci 1991;301:221-30.

3 Wall TC, Peyton RB, Corey GR. Gonococcal endocarditis: a new look at an old disease. Medicine 1989;68:375-80. 